\title{
Atomic Line Shapes in the Presence of an External Magnetic Field
}

M. L. Adams, R. W. Lee, H. A. Scott, H. K. Chung, L. Klein

This article was submitted to $13^{\text {th }}$ Topical Conference on Atomic Processes in Plasma, Gatlinburg, TN., April 22-25, 2002

\section{July 3, 2002}

\section{U.S. Department of Energy}

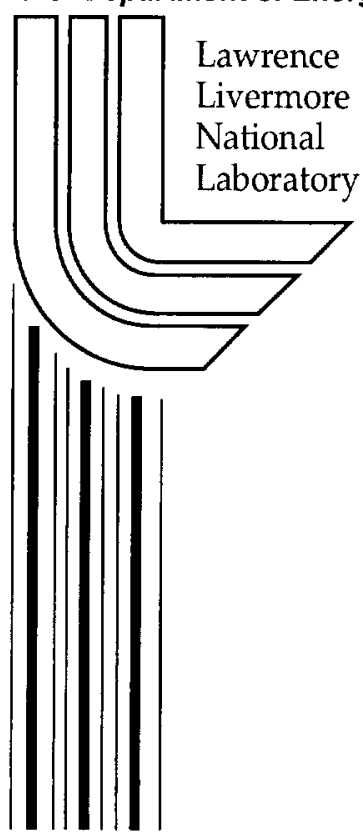




\section{DISCLAIMER}

This document was prepared as an account of work sponsored by an agency of the United States Government. Neither the United States Government nor the University of California nor any of their employees, makes any warranty, express or implied, or assumes any legal liability or responsibility for the accuracy, completeness, or usefulness of any information, apparatus, product, or process disclosed, or represents that its use would not infringe privately owned rights. Reference herein to any specific commercial product, process, or service by trade name, trademark, manufacturer, or otherwise, does not necessarily constitute or imply its endorsement, recommendation, or favoring by the United States Government or the University of California. The views and opinions of authors expressed herein do not necessarily state or reflect those of the United States Government or the University of California, and shall not be used for advertising or product endorsement purposes.

This is a preprint of a paper intended for publication in a journal or proceedings. Since changes may be made before publication, this preprint is made available with the understanding that it will not be cited or reproduced without the permission of the author.

This work was performed under the auspices of the United States Department of Energy by the University of California, Lawrence Livermore National Laboratory under contract No. W-7405-Eng-48.

This report has been reproduced directly from the best available copy.

Available electronically at http://www.doc.gov/bridge

Available for a processing fee to U.S. Department of Energy

And its contractors in paper from

U.S. Department of Energy

Office of Scientific and Technical Information

P.O. Box 62

Oak Ridge, TN 37831-0062

Telephone: (865) 576-8401

Facsimile: (865) 576-5728

E-mail: reports@adonis.osti.gov

Available for the sale to the public from

U.S. Department of Commerce

National Technical Information Service

5285 Port Royal Road

Springfield, VA 22161

Telephone: (800) 553-6847

Facsimile: (703) 605-6900

E-mail: orders@ntis.fedworld.gov

Online ordering: http://www.ntis.gov/ordering.htm

\section{OR}

Lawrence Livermore National Laboratory

Technical Information Department's Digital Library

http://www.llnl.gov/tid/Library.html 


\title{
Atomic line shapes in the presence of an external magnetic field
}

\author{
M.L. Adams*, R.W. Lee, H.A. Scott, H.K. Chung ${ }^{\dagger}$ and L. Klein** \\ ${ }^{*}$ Department of Nuclear Engineering, Massachusetts Institute of Technology, NWI6-230, 167 \\ Albany St., Cambridge, MA 02139, USA \\ ${ }^{\dagger}$ University of California, Lawrence Livermore National Laboratory, P.O. Box 808, Livermore, CA \\ 94551, USA \\ ${ }^{* *}$ Department of Physics and Astronomy, Howard University, Washington, DC 20059, USA
}

\begin{abstract}
Both the theoretical basis and computational approach for extending the capabilities of a spectral line broadening code are presented. Following standard line broadening theory, the effects of an external magnetic field are incorporated into the atomic Hamiltonian and plasma average. In the presence of an external magnetic field the atomic Hamiltonian angular properties are altered - atomic energy levels are perturbed and the spectral emission line is polarized. The magnetic field introduces a preferential axis that changes the plasma average. These extensions have been incorporated into a new spectral line broadening code that is applied to several problems of importance to the understanding of tokamak edge plasmas. Applications fall into two broad categories: 1) determination of local plasma properties from distinct line shape features; and 2) consideration of global plasma phenomenon, such as radiation transport. Observable features of the Zeeman effect make $H_{\alpha}$ a good magnetic field diagnostic. $H_{\beta}$ does not make a good electron density diagnostic since the Zeeman effect is comparable to the Stark effect for a majority of tokamak edge plasma conditions. When optically thick lines exist the details of the spectral line shapes are shown to significantly influence the transport of radiation throughout the system.
\end{abstract}

\section{INTRODUCTION}

Plasma properties in the tokamak edge typically span several orders of magnitude at any given time during a typical experiment $[1,2]$. The system properties span the following range: $10^{12}<n_{e}\left(\mathrm{~cm}^{-3}\right)<10^{16}, 0.1<T_{e}(\mathrm{eV})<500.0$ and $0.1<B(\mathrm{~T})<10.0$; where $n_{e}$ is the electron density, $T_{e}$ is the electron temperature and $B$ is the magnetic field strength (assuming the permeability is one). While this large variation in plasma conditions poses many interesting theoretical and computational challenges, for the present spectral line shape study the high-density low-temperature (HDLT) edge plasma regions are of particular importance [3].

HDLT plasmas are found in detached divertor experiments near the divertor target plate and in MARFEs (an axisymmetric radiating phenomenon that is poloidally localized in the plasma edge). In Alcator C-Mod, HDLT plasma properties are of the order: $n_{e} \sim 10^{15} \mathrm{~cm}^{-3}, T_{e} \sim 1.0 \mathrm{eV}, B \sim 6 \mathrm{~T}$ and $L \sim 5 \mathrm{~cm}$; where $L$ is a characteristic spatial extent of the region. What makes these plasmas especially interesting is that they are partially ionized (there exists a significant fraction of neutral particles in the edge plasma) and they interact strongly with line radiation (optical depths are much greater than unity). Thus, in HDLT plasmas the details of spectral line shapes will be important 
to the spatial and frequency variation of the radiation field, which in turn influences the spatial variation in level populations and ultimately the transport of (non-radiative) energy. It is this connection between energy transport and the details of spectral line shapes that motivates this research.

This paper proceeds as follows. Section 2 begins by reviewing standard line broadening theoretical and computational methods [4]. The effects of an external magnetic field are then systematically incorporated into both the atomic Hamiltonian and the plasma average. The resulting code is named TOTALB. Section 3 applies TOTALB several problems of importance to the understanding of tokamak edge plasmas. Section 4 provides concluding remarks and proposes potential future research directions.

\section{LINE SHAPE FORMALISM}

In this section the pertinent elements of the current embodiment of atomic spectral line shape calculations are discussed. Then effects of an external magnetic field on the plasma average and atomic Hamiltonian are considered. We note that while the atomic Hamiltonian modification is complete, future work is needed to rigorously incorporate magnetic effects into the plasma average.

\subsection{General spectral line shape}

Following Baranger [5] and Kolb and Griem [6], it is customary to begin with the quantum electrodynamical formula for the power radiated by an atom in an electric dipole transition from an initial state $(i)$ to a final state $(f)$ :

$$
\begin{aligned}
P(\omega) & =\frac{4 \omega^{4}}{3 c^{3}} \phi(\omega) \\
\phi(\omega) & =\frac{1}{2 \pi} \int_{-\infty}^{\infty} \Phi(t) e^{i \omega t} d t \\
\Phi(t) & =\sum_{i f} \rho_{i}\left\langle\psi_{i}(0)|\mathbf{d}| \psi_{f}(0)\right\rangle\left\langle\psi_{f}(t)|\mathbf{d}| \psi_{i}(t)\right\rangle
\end{aligned}
$$

where $\phi(\omega)$ is the spectral line shape (normalized to unity $\int \phi(\omega) d \omega=1$ ) and $\Phi(t)$ is the time correlation function. $\sum_{i f}$ represents a sum over all final states and an average over all initial states that contribute to the line shape, $\rho_{i}$ is the initial density matrix of the system, $|\psi(t)\rangle$ represents the state (in the Schrödinger picture) of the system and $\mathbf{d}$ is the time-independent electric dipole moment of the system. The quantity $\frac{4 \omega^{4}}{3 c^{3}}$ can be added after the calculation of $\phi(\omega)$ since the frequency variation of $\omega^{4}$ is small compared to $\phi(\omega)$; this transfers the focus from the power radiated to the spectral line shape. To connect with dynamical theories the initial focus is further transferred to $\Phi(t)$.

Following Fano [7, 8], the correlation function can be simplified by first using general operator techniques and then separating an emitting atom (a) from its surrounding 
plasma $(p)$. This allows the general correlation function to be written in the form:

$$
\Phi(t)=\operatorname{Tr}_{a}\left[\mathbf{d} \exp \left\{-\frac{i}{\hbar}\left(L^{a}-i \Gamma\right) t\right\} \rho_{i}^{a} \mathbf{d}\right]_{A v}
$$

where $L^{a}$ is the Liouville operator pertaining to the atomic Hamiltonian, $\Gamma$ is the relaxation theory collisional operator, $\rho^{a}$ is the initial atomic density matrix, the trace is performed over all atomic states and the average $(A v)$ is over plasma states.

Following Griem [9], the spectral line shape calculation considers those electric dipole transitions between bound states that contribute to a well defined frequency range. The associated atomic states are grouped into an upper manifold (with states $|\alpha\rangle$ ) and a lower manifold (with states $|\beta\rangle$ ). Dipole transitions between states of the same manifold and collisional transitions between different manifolds are not considered. The plasma average is handled by simultaneously introducing an electron model and an ion microfield model into the correlation function:

$$
\Phi(t)=\int P(E)\left\langle\left\langle\alpha \beta\left|\mathbf{d} \exp \left\{-\frac{i}{\hbar}\left(L_{E}^{a}-i \Gamma\right) t\right\} \rho_{i}^{a} \mathbf{d}\right| \alpha^{\prime} \beta^{\prime}\right\rangle\right\rangle d E
$$

where $P(E)$ is the quasi-static electric ion microfield distribution function, $L_{E}^{a}$ is the Liouville operator pertaining to the atomic Hamiltonian for a particular ionic field $(E)$, $\Gamma$ is now the electron collision operator and $|\alpha \beta\rangle\rangle$ represents the atomic state in Liouville (or line) space. Regardless of the specific models used in the plasma average there exists a general computational approach for solving this problem.

Following Calisti et al. [4], the spectral line shape computational approach centers around solving the complex eigenvalue problem for a fixed ionic field and then averaging over possible ionic fields. Since the correlation function must be real, which requires that $\phi(-\omega)=\phi^{*}(\omega)$, the line shape equation can be written:

$$
\begin{aligned}
\phi(\omega) & =\frac{1}{\pi} \operatorname{Re} \int_{0}^{\infty} \Phi(t) e^{i \omega t} d t \\
& =\int P(E) \frac{1}{\pi} \operatorname{Re} \int_{0}^{\infty}\left\langle\left\langle\alpha \beta\left|\mathbf{d} \exp \left\{-\frac{i}{\hbar}\left(L_{E}^{a}-i \Gamma\right) t\right\} \rho_{i}^{a} \mathbf{d}\right| \alpha^{\prime} \beta^{\prime}\right\rangle\right\rangle e^{i \omega t} d t d E \\
& =\sum_{j=1}^{n_{E}} W_{j} \sum_{k} \frac{c_{1 k}\left[\omega-x_{k}\left(E_{j}\right)\right]+c_{2 k} y_{k}\left(E_{j}\right)}{\left[\omega-x_{k}\left(E_{j}\right)\right]^{2}+y_{k}\left(E_{j}\right)^{2}}
\end{aligned}
$$

where the integration over $E$ has been replaced by a sum that represents an average over discrete ionic fields with weights given by $W_{j}$. Let $\left.|k\rangle\right\rangle$ be the basis in which $L_{E_{j}}^{a}-i \Gamma$ is diagonal, obtained through use of a transformation matrix $T_{E_{j}}$, with complex eigenvalues $z_{k}\left(E_{j}\right)=x_{k}\left(E_{j}\right)+i y_{k}\left(E_{j}\right)$. The coefficients $c_{k 1}$ and $c_{k 2}$ depend on $\mathbf{d}$ and $T_{E_{j}}$. In order to implement this formula atomic structure information is needed.

An optimal set of atomic data is obtained by using the fact that atomic structure information only enters in the selection of states that contribute to radiation in a specific frequency range and the evaluation of the Liouville operator matrix elements. In Liouville space, or line space, the Liouville operator matrix elements are:

$$
\begin{aligned}
\left\langle\left\langle\alpha \beta\left|L_{E}\right| \alpha \beta\right\rangle\right\rangle & =\hbar \omega_{\alpha \beta} \\
\left\langle\left\langle\alpha \beta\left|L_{E}\right| \alpha \beta^{\prime}\right\rangle\right\rangle & =\left\langle\beta|\mathbf{d}| \beta^{\prime}\right\rangle E \\
\left\langle\left\langle\alpha \beta\left|L_{E}\right| \alpha^{\prime} \beta\right\rangle\right\rangle & =-\left\langle\alpha|\mathbf{d}| \alpha^{\prime}\right\rangle E
\end{aligned}
$$


where $\hbar \omega_{\alpha \beta}$ is the energy difference between states $|\alpha\rangle$ and $|\beta\rangle,\langle\beta|\mathbf{d}| \alpha\rangle$ is the electric dipole matrix element in Hilbert space and $E$ is the ionic field strength. Thus, numerically speaking, the atomic data information must include: 1) an index to label each state; 2) quantum information ascribable to each state; and 3) electric dipole matrix elements between states.

From the diagonal element of the Liouville operator matrix elements, it is clear that energy level information is needed to describe each transition; this information is also needed for the selection of states mentioned above. Regarding the electric dipole matrix elements, in their current form they require complete specification of the relevant quantum numbers of the states involved. Due to the spherical symmetry properties of atoms with complex atomic structure the matrix elements can be simplified by using the Wigner-Eckart theorem [10] as follows:

$$
\left\langle\gamma J M\left|\mathbf{d}_{q}^{(1)}\right| \gamma J^{\prime} M^{\prime}\right\rangle=(-1)^{J-M}\left(\begin{array}{ccc}
J & 1 & J^{\prime} \\
-M & q & M^{\prime}
\end{array}\right)\left\langle\gamma J \| \mathbf{d}^{(1)}|| \gamma^{\prime} J^{\prime}\right\rangle
$$

where tensor-operator notation has been explicitly used, the second term on the righthand-side (RHS) is a Wigner 3-j symbol and the square of the last reduced matrix element on the RHS is known as the electric dipole line strength. The electric dipole line strength includes radial matrix information and is independent of angular momentum coupling schemes - a detail that allows atomic structure codes based on either LS or JJ angular momentum coupling schemes to generate data for the line shape code. Now the only atomic data necessary to calculate the electric dipole matrix elements are the $2 J+1$ value of each state, which specifies the possible orientations of the 3-j symbol, and the reduced matrix elements between states.

\subsection{Plasma average modifications}

The plasma average consists of two parts: the electron collision operator, which is calculated within the framework of binary collision relaxation theory, and the quasistatic electric ion microfield. An external magnetic field introduces a preferential axis into the system that destroys the arbitrary orientation of the electric dipole operator and can alter the dynamical properties of the plasma.

The quasi-static ion microfield model assumes that the emitting atom is surrounded by a spherically symmetric plasma. The electric dipole moment can be chosen to point in an arbitrary direction and the ion microfield distribution must be a function only of ionic field strength. With the introduction of a preferential axis, the spherical symmetry is broken and the integration over the microfield distribution becomes anisotropic:

$$
P(E) d E \rightarrow P^{\prime}\left(E_{\perp}, E_{\|}\right) d E_{\perp} d E_{\|}
$$

where the subscripts refer to the direction of the magnetic field. This modification amounts to an average over electric field directions relative to the magnetic axis. The modification increases computational time by introducing a double integration into the plasma average and tripling the number of non-zero electric dipole matrix elements 
that enter the complex eigenvalue problem. Note that while this modification to the microfield integration has been made, the microfield itself is assumed unchanged by the introduction of a magnetic field. The following discussion considers the limitations of this model.

Ion dynamics might be important when an external magnetic field is introduced into the spectral line shape formalism. The quasi-static ion microfield model is valid provided the half-width at half-maximum (HWHM) of the normalized line profile $\left(\Delta \omega_{1 / 2}\right)$ is much greater than the ion plasma frequency $\left(\omega_{p i}\right)$. In the presence of a magnetic field the Lorentz force $(q \mathbf{v} \times \mathbf{B})$ increases the plasma frequency and decreases the region of validity of the quasi-static ion microfield model [11]. Thus, a more general criterion for neglecting ion dynamics, which accounts for the asymmetry of the ion plasma frequency, is:

$$
\Delta \omega_{1 / 2} \gg \omega_{L H} \geq \omega_{p i}
$$

where the lower hybrid resonant frequency $\left(\omega_{L H}\right)$ is the ion plasma frequency perpendicular to the magnetic field. If ion dynamics is important then anisotropy should be considered further.

The electron collision operator is valid provided $\Delta \omega_{1 / 2}$ is much less than the electron plasma frequency $\left(\omega_{p e}\right)$. In the presence of a magnetic field the Lorentz force increases the plasma frequency and increases the region of validity of the electron collision operator. The general condition for the validity of the electron collision operator can be expressed as:

$$
\Delta \omega_{1 / 2} \ll \omega_{p e} \leq \omega_{U H}
$$

where the hybrid resonant frequency $\left(\omega_{U H}\right)$ is the electron plasma frequency perpendicular to the magnetic field. While the external magnetic field strengthens the condition it also introduces anisotropy.

The consideration of magnetic effects on the motion of charged particles results in a constraint on the binary collision relaxation model straight-line path (SLP) approximation. The condition for validity of the SLP approximation is that the Larmor radius must be greater than the Debye length $\left(r_{L}>\lambda_{D}\right)$; or similarly the Larmor frequency must be greater than the electron plasma frequency $\left(\omega_{L}=\frac{q B}{2 m_{e}}<\omega_{p e}\right)$. Explicitly writing the plasma property dependence involved in this condition places an upper limit on the magnetic field strength: $B(T)<4.5 \sqrt{n_{e 14}}$, where $n_{e 14}$ is in units of $10^{14} \mathrm{~cm}^{-3}$. Thus, for most HDLT edge plasma phenomenon the SLP approximation is valid.

\subsection{Atomic Hamiltonian modification}

In the presence of an external magnetic field the atomic Hamiltonian angular properties are altered - atomic energy levels are perturbed and the line shape is polarized. We use the non-relativistic one-electron atom Hamiltonian to illustrate this modification and at the same time introduce the approximations employed. Generalization to atoms with complex structure is then accomplished through the use of Racah algebra. 


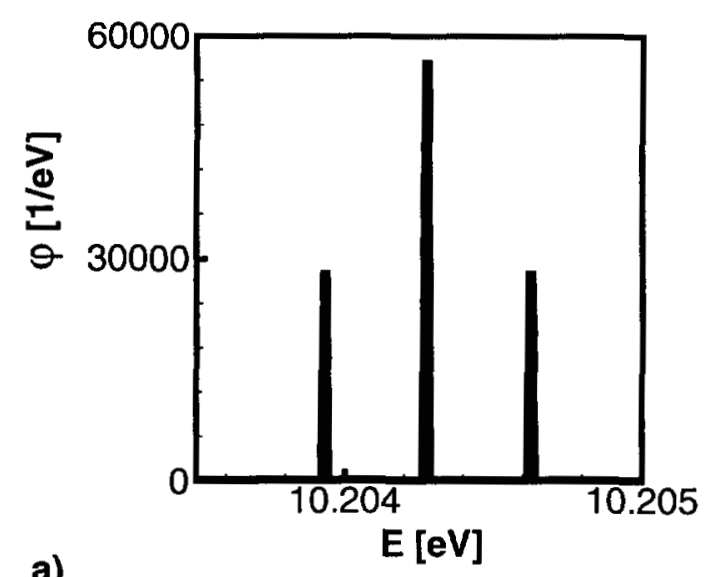

a)

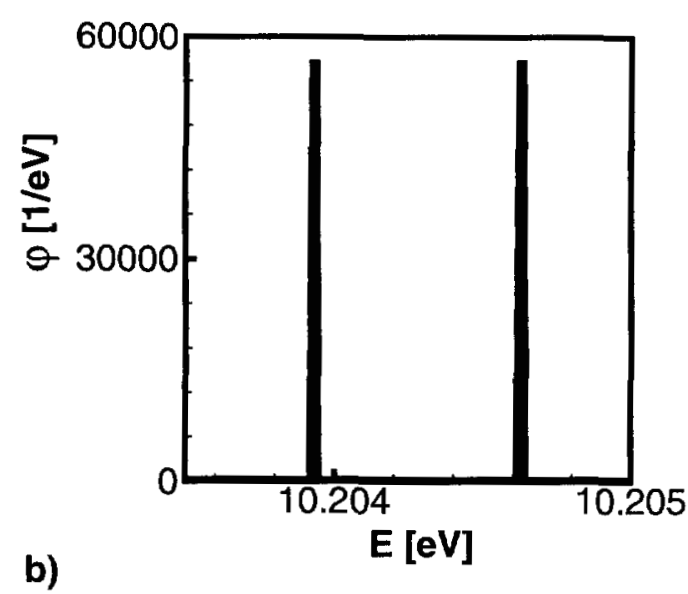

FIGURE 1. Zeeman components of Hydrogen $L y_{\alpha}$ in a $B=6 \mathrm{~T}$ magnetic field. The observation direction is a) perpendicular to the magnetic axis $(\beta=\pi / 2)$ and $b)$ along the magnetic axis $(\beta=0)$.

The atomic Hamiltonian for a one-electron atom in a uniform external magnetic field, with vector potential $\mathbf{A}=-\frac{1}{2} \mathbf{R} \times \mathbf{B}$, is given by:

$$
\begin{aligned}
H & =\frac{1}{2 m_{e}}[\mathbf{P}-q \mathbf{A}(\mathbf{R})]^{2}+V(\mathbf{R}) \\
& =\left\{\frac{P^{2}}{2 m_{e}}+V(\mathbf{R})\right\}-\frac{\mu_{B}}{\hbar} \mathbf{L} \cdot \mathbf{B}+\frac{q^{2} B^{2}}{8 m_{e}} R_{\perp}^{2}
\end{aligned}
$$

where $\mu_{B}=\frac{q \hbar}{2 m_{e}}$ is the Bohr magneton, $\mathbf{L}=\mathbf{R} \times \mathbf{B}$ and $R_{\perp}^{2}=R^{2}-\frac{(\mathbf{R} \cdot \mathbf{B})^{2}}{B^{2}}$ is the projection of $\mathbf{R}$ onto the plane perpendicular to $\mathbf{B}$ [12]. The term proportional to $B$ is called the paramagnetic term; the term proportional to $B^{2}$ is called the diamagnetic term. Assuming $B$ is small $\left(B<10^{3} \mathrm{~T}\right)$ the following energy ordering applies:

$$
\frac{\Delta E_{1}}{\Delta E_{0}} \sim \frac{\Delta E_{2}}{\Delta E_{1}} \ll 1
$$

where the subscript denotes order with respect to $B$. Such an energy ordering is valid for magnetic confinement fusion, where $B<10 \mathrm{~T}$, and can be written in terms of plasma properties as:

$$
\mu_{B} B=\hbar \omega_{L} \ll E_{I}=\frac{1}{2} \alpha^{2} m_{e} c^{2}
$$

where $\alpha$ is the fine structure constant. Thus, the diamagnetic term is negligible compared to the paramagnetic term and will not be considered.

The change in frequency and polarization of the emitted radiation due to the paramagnetic term is collectively called the Zeeman effect. These effects are illustrated in Fig. 1. The line shapes depicted in Fig. 1 were calculated with TOTALB using non-relativistic atomic data and neglecting both plasma broadening and Doppler broadening. The transition energies and relative magnitudes agree with first order perturbation theory [12]. 
For atoms with complex atomic structure, the paramagnetic term only affects angular properties of the atom. The general paramagnetic term can be written as:

$$
\mathbf{L} \cdot \mathbf{B} \rightarrow\left[\mathbf{J}+\left(g_{s}-1\right) \mathbf{S}\right] \cdot \mathbf{B}
$$

where $g_{s} \cong 2.00232$ is the anomalous gyromagnetic ratio for electron spin. Now the complete atomic Hamiltonian can be written as:

$$
H=H_{0}+H_{S}+H_{Z}
$$

where $H_{0}$ represents the full complex atomic Hamiltonian (that may include fine structure effects); $H_{S}$ (Stark) and $H_{Z}$ (Zeeman) are given by:

$$
\begin{aligned}
& H_{S}=-\mathbf{E} \cdot \mathbf{d}^{(1)} \\
& H_{Z}=-\mathbf{B} \cdot \mu^{(1)}=\mu_{B} \mathbf{B} \cdot\left[\mathbf{J}+\left(g_{s}-1\right) \mathbf{S}\right]
\end{aligned}
$$

To evaluate the paramagnetic matrix element let $\mathbf{B}=B \hat{z}$ and $g_{s}=2$. The matrix element takes the form:

$$
\frac{1}{\mu_{B} B}\left\langle\gamma J M\left|H_{Z}\right| \gamma^{\prime} J^{\prime} M^{\prime}\right\rangle=\left\langle\gamma J M\left|\mathbf{J}_{0}^{(1)}+\mathbf{S}_{0}^{(1)}\right| \gamma J^{\prime} M^{\prime}\right\rangle
$$

In a manner similar to the electric dipole matrix element this equation can be simplified by application of the Wigner-Eckart theorem:

$$
\left\langle\gamma J M\left|\mathbf{J}_{0}^{(1)}+\mathbf{S}_{0}^{(1)}\right| \gamma^{\prime} J^{\prime} M^{\prime}\right\rangle=(-1)^{J-M}\left(\begin{array}{ccc}
J & 1 & J^{\prime} \\
-M & 0 & M^{\prime}
\end{array}\right)\left\langle\gamma J\left\|\mathbf{J}^{(1)}+\mathbf{S}^{(1)}\right\| \gamma^{\prime} J^{\prime}\right\rangle
$$

Analogous to the electric dipole line strength the square of the reduced matrix element on the RHS is called the magnetic dipole line strength. Note that the magnetic dipole line strength also includes radial matrix information and is not dependent on an angular momentum coupling scheme.

\section{TOKAMAK APPLICATIONS}

Applications of TOTALB fall into two categories: 1) determination of local plasma properties from distinct line shape features; and 2) consideration of global plasma phenomenon, such as particle and energy transport. This section considers both types of applications. In the numerical calculations that follow we use atomic data from the relativistic complex atomic structure code HULLAC (Hebrew University Lawrence Livermore Atomic Code) [13].

\subsection{Hydrogen resonance line}

In HDLT plasmas the resonance line of Hydrogen $\left(L y_{\alpha}\right)$ has a significant optical depth $(\tau \sim \chi L>1$, where $\chi$ is the line radiation absorption coefficient and $L$ is a length 
characterizing the extent of the plasma region). Since details of the spectral line shape will affect the plasma opacity, it is interesting to quantify magnetic field effects on $\tau$.

To begin, the line radiation absorption coefficient for a plasma in local thermodynamic equilibrium (LTE) is [14]:

$$
\begin{aligned}
\chi(v) & =n_{i} B_{i j} \frac{h v_{i j}}{4 \pi} \phi_{i j}(v)\left[1-\exp \left\{-\frac{h v_{i j}}{T}\right\}\right] \sim n_{i}\left\{\frac{e^{2}}{4 \pi \varepsilon_{0}} \frac{\pi}{m c} \phi_{i j}(v)\right\} f_{i j} \\
\chi_{0} & \sim n_{i 14}\left\{\frac{0.011}{\Delta E_{e V}}\right\} f_{i j}\left[\mathrm{~cm}^{-1}\right]
\end{aligned}
$$

where $i(j)$ refers the lower (upper) level of a bound-bound transition, $B$ is the Einstein B coefficient, $h v_{i j}$ is the line center energy, $\phi$ is the line profile, $T$ is the thermodynamic temperature, $f$ is the line absorption oscillator strength, $\chi_{0}$ is the line center absorption coefficient and $\Delta E$ is the full-width at half-maximum (FWHM) of the line shape in units of electron-Volts (eV). In Eqs. 26 and 27 the terms in curly braces represent an effective cross section for photon absorption. The latter equation clearly shows that $\Delta E$ and $\chi_{0}$ are inversely proportional; as $\Delta E$ increases the cross section decreases and hence $\chi_{0}$ decreases. That is, as a line shape becomes broader the line absorption oscillator strength is distributed over a greater frequency range. With $\chi_{0}$ given by Eq. 27 the optical depth can be estimated with a suitable choice of $L$.

Now the effect of an external magnetic field on opacity can be quantified by using TOTALB. As an example, we consider the Hydrogen $L y_{\alpha}$ line shape for an HDLT plasma $\left(n_{e}=10^{15} \mathrm{~cm}^{-3}\right.$ and $\left.T_{e}=1 \mathrm{eV}\right)$ with $(B=6 \mathrm{~T})$ and without $(B=0 \mathrm{~T})$ an external magnetic field. As the magnetic field increased from $B=0 \mathrm{~T}$ to $6 \mathrm{~T}$ the FWHM increased from $\Delta E=0.0009 \mathrm{eV}$ to $0.0011 \mathrm{eV}$. Setting $L=1 \mathrm{~cm}$ and choosing a ground state density of $n_{1}=10^{14} \mathrm{~cm}^{-3}$ yields values without (with) magnetic effects: an absorption coefficient of $5.1 \mathrm{~cm}^{-1}\left(4.2 \mathrm{~cm}^{-1}\right)$; effective photon mean free path of $0.20 \mathrm{~cm}(0.24 \mathrm{~cm})$; an optical depth of 5.1 (4.2). Thus, magnetic line broadening reduced the optical depth by approximately $18 \%$.

\subsection{Magnetic field diagnostic}

In Eq. 21 there are three terms on the RHS: $H_{0}$ pertains to the energy of a state; $H_{S}$ leads to the Stark effect that is proportional to the plasma density as well as the principle quantum number (which scales approximately as $n^{2}$ ) [10]; and, $H_{Z}$ leads to the Zeeman effect and is proportional to $\mathbf{B}$. Thus, the shift and intensity of a spectral line shape component are dependent on the relative contributions of both the Stark effect and the Zeeman effect. Furthermore, the intensity of a component will vary with angle of observation relative to the magnetic axis due to the polarization properties of the Zeeman effect. From these line shape features, if the Zeeman effect dominates the Stark effect then both the magnetic field strength and angle of the magnetic field relative to the direction of observation can be accurately determined using TOTALB.

For HDLT plasmas in the Alcator C-Mod tokamak $H_{\alpha}$ is a prime magnetic field diagnostic candidate. High-resolution $H_{\alpha}$ measurements are made routinely in Alcator C-Mod [15] and the data clearly exhibit the Zeeman effect signature [3]. While these 
measurements were intended to study the Hydrogen to Deuterium ratio and plasma fluctuations, they are ideally suited for magnetic field diagnostic purposes.
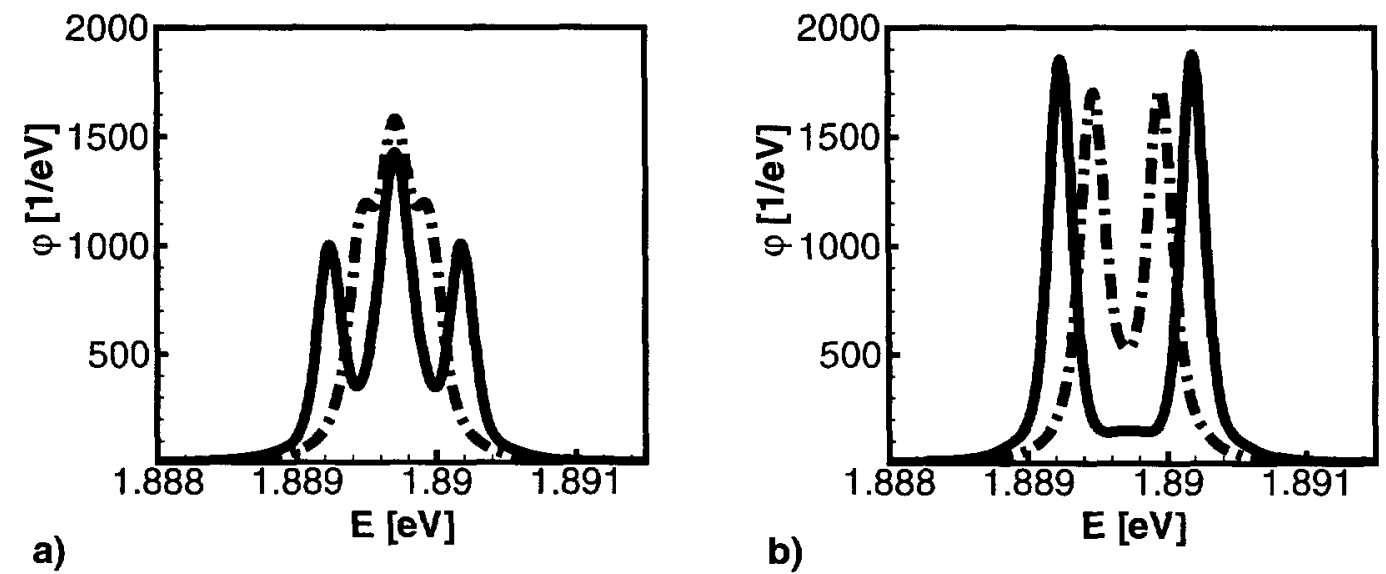

FIGURE 2. a) Magnetic field strength variation from $B=4 \mathrm{~T}$ (dash-dot line) to $B=8 \mathrm{~T}$ (solid line). Plasma conditions are: $n_{e}=10^{15} \mathrm{~cm}^{-3}$ and $T_{e}=1 \mathrm{eV}$. The angle of observation is perpendicular to the magnetic axis $(\beta=\pi / 2)$. b) Same plasma conditions as in (a) but now the angle of observation is along the magnetic axis $(\beta=0)$.

To demonstrate the magnetic field diagnostic procedures, we consider first the determination of the magnetic field strength. Figure 2a shows the sensitivity of $H_{\alpha}$ to changes in magnetic field strength for conditions typical of an HDLT plasma. For this line shape, calculated for an angle of observation perpendicular to the magnetic axis, determination of the magnetic field strength is performed by numerically matching the energy difference between the central $\pi$-component and a $\sigma$-component. This is not the same as inferring the magnetic field from the first-order Zeeman effect alone $\left(\Delta E_{1 / 2}=\mu_{B} B\right)$ since this would be valid only in the absence of plasma broadening. To quantify the error made by neglecting plasma broadening, the magnetic field strength calculated using only the first-order Zeeman formula with $\Delta E_{1 / 2}$ taken from the $B=8 \mathrm{~T}$ curve in Fig. 2a would be 8.21T. In this case the result is an overestimate of the magnetic field strength by $0.21 \mathrm{~T}$ - resulting in a significant difference in the inferred emission region in tokamaks.

Figure. $2 b$ shows the line shape from an angle of observation along the magnetic axis, with the same plasma conditions as in Fig. 2a, and reveals a better candidate for determining magnetic field strength. The absence of the central $\pi$-component allows the diagnostic to be applied for lower magnetic field tokamaks. Figure $2 b$ also illustrates the variation in intensity, but not shift, of the line profile components with angle of observation relative to the magnetic axis. It is this feature that can be used to determine $\beta$ by numerically matching the relative intensity of the $\pi$-component to a $\sigma$-component.

\subsection{Electron density diagnostic}

In plasmas without an external magnetic field the spectral line width is commonly used to determine plasma densities [16]. For instance, Griem recommends $H_{\beta}$ as an ex- 
cellent candidate for determining the electron density in plasmas where $n_{e}>10^{14} \mathrm{~cm}^{-3}$. In this density regime the linear Stark effect dominates over other broadening mechanisms and the FWHM scales as $n_{e}^{2 / 3}$. Below an electron density of $10^{14} \mathrm{~cm}^{-3}$ fine structure effects begin to enter and the dominant FWHM dependence makes a transition from the linear Stark effect to quadratic ion impact [17].

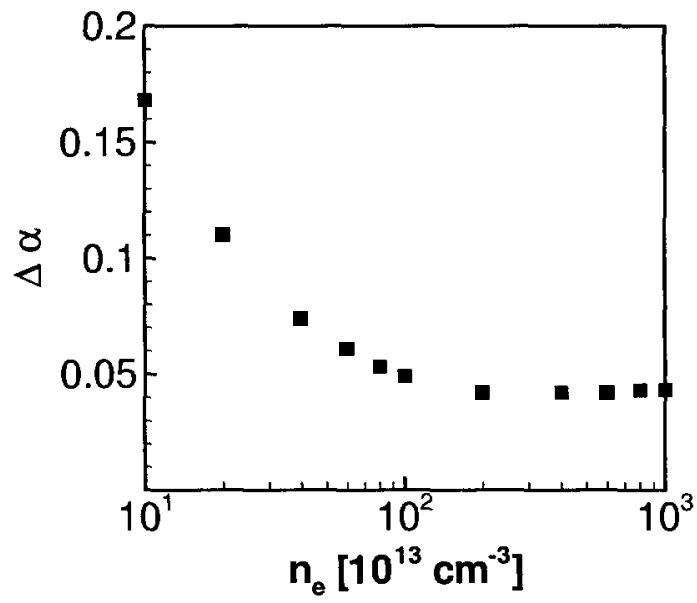

FIGURE 3. A plot of the $H_{\beta}$ HWHM including the $n_{e}^{2 / 3}$ scaling $\left(\Delta \alpha=\Delta \lambda_{1 / 2} / n_{e}^{2 / 3}\right)$ as a function of electron density. The curve asymptotes when the linear Stark effect dominates other broadening mechanisms. The plasma properties are: $B=6 \mathrm{~T}, T_{e}=2 \mathrm{eV}$ and $\beta=\pi / 2$.

In plasmas with an external magnetic field the Stark effect competes with the Zeeman effect. Günter-Könies [18] has investigated the feasibility of $H_{\beta}$ as a density diagnostic for low magnetic field tokamaks $(B \sim 2 \mathrm{~T})$ and found that it is useful provided $n_{e}>$ $2 \times 10^{14} \mathrm{~cm}^{-3}$. Figure 3, which was calculated for conditions typical of Alcator C-Mod $(B=6 \mathrm{~T})$, indicates that $H_{\beta}$ is not useful as an electron density diagnostic unless $n_{e}>$ $2 \times 10^{15} \mathrm{~cm}^{-3}$. Future density diagnostic efforts would have better success considering high- $n$ lines that take advantage of the $n^{2}$ Stark scaling, e.g., $H_{8 \rightarrow 2}$.

\subsection{Finite plasma slab}

HDLT tokamak edge plasmas strongly interact with hydrogen line radiation. Thus, in HDLT plasmas the details of spectral line shapes will determine the spatial and frequency variation of opacity, which in turn influences the spatial variation in level populations and ultimately the transport of energy. Through full integration of TOTALB into a coupled non-local thermodynamic (NLTE) atomic kinetics and radiation transfer code CRETIN [19], the effects of magnetically broadened line shapes can be quantified.

We consider a one-dimensional plasma slab in the $x$-y plane with a thickness of $1 \mathrm{~cm}$ and plasma properties: $\mathbf{B}=6 \hat{x} T, T_{e}=1.0 \mathrm{eV}, n_{e}=10^{15} \mathrm{~cm}^{-3}$ and $n_{1}=10^{14} \mathrm{~cm}^{-3}$ (ground state neutral hydrogen density). Figure 4 a demonstrates the $L y_{\alpha}$ emissivity variation with angle relative to the magnetic axis. Figure $4 \mathrm{~b}$ plots the spectral radiation intensity escaping the finite plasma slab with (solid line) and without (dotted line) magnetic field 


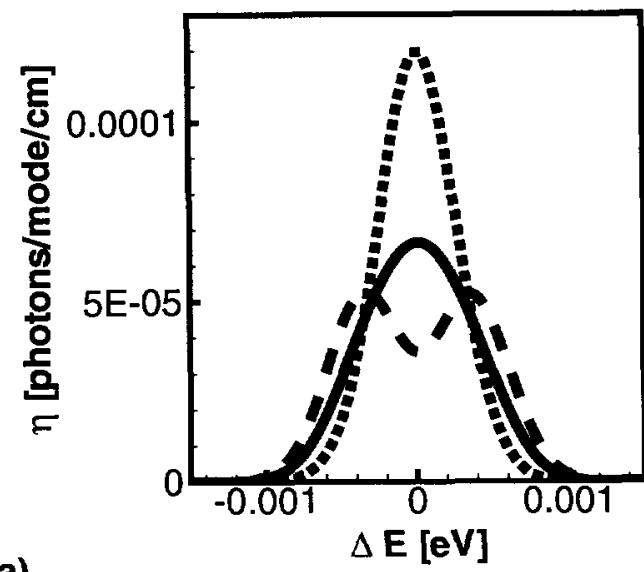

a)

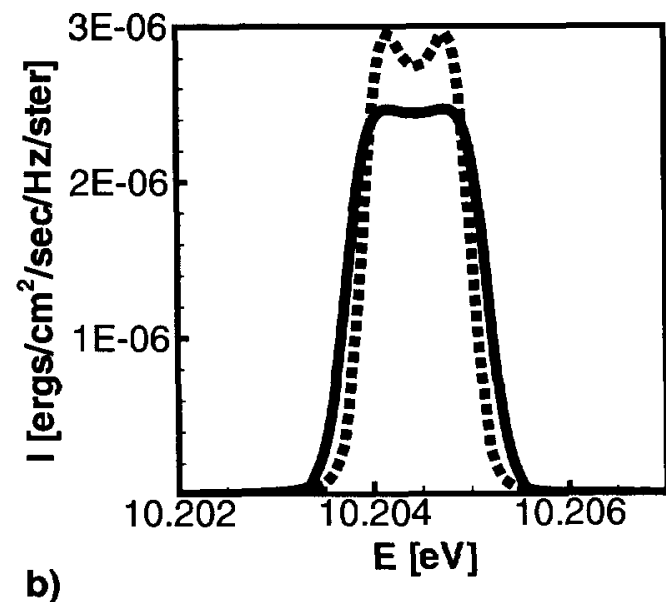

b)

FIGURE 4. a) $L y_{\alpha}$ emissivity at the center of the plasma slab. The solid curve $(\beta=\pi / 2)$ and the dashed curve $(\beta=0)$ are magnetically broadened lines shapes; the dotted line is without magnetic field effects included. b) $L y_{\alpha}$ spectral radiation intensity escaping the finite plasma slab with (solid line) and without (dotted line) magnetic field effects.

effects. The integrated intensity increased by approximately $10 \%$ - this difference will increase with optical depth. Magnetic field effects will not directly affect the integrated intensity of optically thin lines. All the emitted photons in an optically thin line escape and their distribution relative to line center is irrelevant. However, optically thick lines can affect the excited state populations leading to changes in emission from optically thin lines, as well as ionization, recombination and energy loss rates [20].

\section{DISCUSSION}

The effects of an external magnetic field have been added to the spectral line broadening model TOTAL [4] and the goal of producing a numerically fast magnetically broadened line shape model for inclusion into large transport codes has been reached. However, while the atomic Hamiltonian modifications are complete, future work in needed to rigorously incorporate magnetic effects into the plasma average.

Since the region of validity of the quasi-static ion microfield is restricted by anisotropic ion dynamics, future research is needed to determine the role of $P\left(E_{\|}, E_{\perp}\right)$, the conditional probability, in regimes where ion dynamics may become important. Since the SLP approximation is out of the limit of validity, research is needed to look at anisotropic electron collision dynamics.

While these areas of research limit the general applicability of TOTALB, there still exist numerous applications to tokamaks edge plasmas, as well as other systems in plasma physics, condensed matter physics and astrophysics. For tokamak applications, simulation of atomic spectral lines, such as carbon and boron, is now possible and can produce numerous direct diagnostic techniques. More interesting to next generation fusion experiments is the potential to quantify radiation effects on edge plasma transport. 


\section{ACKNOWLEDGMENTS}

MLA would like to thank A.S. Wan (LLNL) and J.P. Freidberg (MIT) for their valuable contributions.

This work was performed under the auspices of the U.S. Department of Energy by the University of California, Lawrence Livermore National Laboratory through contract number W-7405-Eng-48.

\section{REFERENCES}

1. Pitcher, C. S., and Stangeby, P. C., Plasma Physics and Controlled Fusion, 39, 779 (1997).

2. ITER Physics Basis, Nuclear Fusion, 39, 2391 (1999).

3. Adams, M. L., Scott, H. A., Lee, R. W., Terry, J. L., Marmar, E. S., Lipschultz, B., Yu. Pigarov, A., and Freidberg, J. P., JQSRT, 71, 115 (2001).

4. Calisti, A., Khelfaoui, F., Stamm, R., Talin, B., and Lee, R. W., Phys. Rev. A, 42, 5433 (1990).

5. Baranger, M., Phys. Rev., 111, 494 (1958).

6. Kolb, A. C., and Griem, H. R., Phys. Rev., 111, 514 (1958).

7. Fano, U., Rev. Mod. Phys., 29, 74 (1957).

8. Fano, U., Phys. Rev., 131, 259 (1963).

9. Griem, H. R., Spectral Line Broadening by Plasmas, Academic Press, New York, 1979.

10. Cowan, R. D., The Theory of Atomic Structure and Spectra, University of California Press, Berkeley, 1981.

11. Chen, F. F., Introduction to Plasma Physics and Controlled Fusion, Plenum Press, New York, 1984, 2 edn.

12. Cohen-Tannoudji, C., Diu, B., and Laloë, F., Quantum Mechanics, John Wiley \& Sons, New York, 1977.

13. Bar-Shalom, A., Klapisch, M., and Oreg, J., JQSRT, 71, 169 (2001).

14. Mihalas, D., Stellar Atmospheres, W.H. Freeman and Company, New York, 1978, 2 edn.

15. Marmar, E. S., Zweben, S. J., Fulghum, S., and Rostler, P. S., Rev. Sci. Instrum., 70, 1 (1999).

16. Griem, H. R., Principles of Plasma Spectroscopy, Cambridge University Press, New York, 1997.

17. Kelleher, D. E., Wiese, W. L., Helbig, V., Greene, R. L., and Oza, D. H., Physica. Scripta., T47, 75 (1993).

18. Günter, S., and Könies, A., JQSRT, 62, 425 (1999).

19. Scott, H. A., JQSRT, 71, 689 (2001).

20. Adams, M. L., and Scott, H. A., Contrib. Plasma Phys., 42, 395 (2002). 